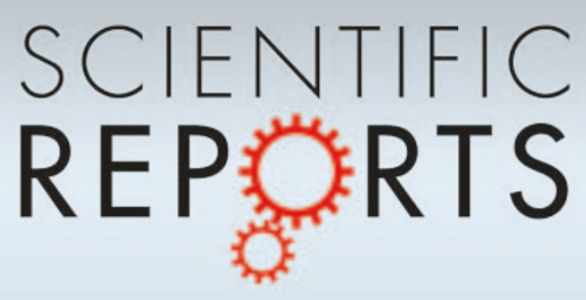

OPEN

SUBJECT AREAS:

MATERIALS SCIENCE

CARBON NANOTUBES AND

FULLERENES

ELECTRONIC MATERIALS AND

DEVICES

NANOTECHNOLOGY

Received

6 March 2012

Accepted

26 March 2012

Published

16 April 2012

Correspondence and requests for materials should be addressed to J.L. (jlou@rice.edu) or H.L. (Hong-lin@ tsinghua.edu.cn)

* These authors contributed equally to this work.

\section{High Electrocatalytic Activity of Vertically Aligned Single-Walled Carbon Nanotubes towards Sulfide Redox} Shuttles

Feng Hao * , Pei Dong ${ }^{2 *}$, Jing Zhang ${ }^{2}$, Yongchang Zhang ${ }^{1}$, Phillip E. Loya ${ }^{2}$, Robert H. Hauge ${ }^{3}$, Jianbao Li ${ }^{4}$, Jun Lou² \& Hong Lin'

\begin{abstract}
'State Key Laboratory of New Ceramics \& Fine Processing, Department of Material Science and Engineering, Tsinghua University, Beijing 100084, P. R. China, ${ }^{2}$ Mechanical Engineering and Materials Science, Rice University, 6100 Main St, Houston, Texas 77005, USA, ${ }^{3}$ The Richard E. Smalley Institute for Nanoscale Science and Technology, Rice University, 6100 Main St, Houston, Texas 77005, USA, ${ }^{4}$ College of Materials Science and Chemical Engineering, Hainan University, Haikou 570228, P. R. China.
\end{abstract}

Vertically aligned single-walled carbon nanotubes (VASWCNTs) have been successfully transferred onto transparent conducting oxide glass and implemented as efficient low-cost, platinum-free counter electrode in sulfide -mediated dye-sensitized solar cells (DSCs), featuring notably improved electrocatalytic activity toward thiolate/disulfide redox shuttle over conventional Pt counter electrodes. Impressively, device with VASWCNTs counter electrode demonstrates a high fill factor of 0.68 and power conversion efficiency up to $5.25 \%$, which is significantly higher than 0.56 and $3.49 \%$ for that with a conventional Pt electrode. Moreover, VASWCNTs counter electrode produces a charge transfer resistance of only $21.22 \Omega$ towards aqueous polysulfide electrolyte commonly applied in quantum dots-sensitized solar cells (QDSCs), which is several orders of magnitude lower than that of a typical Pt electrode. Therefore, VASWCNTs counter electrodes are believed to be a versatile candidate for further improvement of the power conversion efficiency of other iodine-free redox couple based DSCs and polysulfide electrolyte based QDSCs.

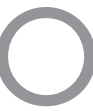

ne of the greatest challenges in the twenty-first century is undoubtedly the energy conversion and storage $^{1}$. In response to the urgent renewable energy needs of modern society and emerging ecological concerns, it is now essential to develop novel inexpensive and environmentally friendly energy conversion and storage systems. Mesoscopic dye-sensitized solar cells (DSCs) have recently emerged as a credible alternative to solid-state $\mathrm{p}-\mathrm{n}$ junction photovoltaic devices, primarily due to its potential to generate electricity on resource-abundant raw materials and energy-saving device processing ${ }^{2}$. Currently, certified power conversion efficiency (PCE) up to $11 \%$ has been achieved in conjunction with a polypyridyl ruthenium complexes based sensitizer and an organic solvent containing $\mathrm{I}^{-} / \mathrm{I}_{3}{ }^{-}$redox couple ${ }^{3}$. Nevertheless, there are substantial drawbacks of this benchmark $\mathrm{I}^{-} / \mathrm{I}_{3}{ }^{-}$redox shuttle hindering its practical applications ${ }^{4}$, such as large energy loss of dye regeneration process, visible light absorption and corrosiveness towards most current-collecting metal grids. Clearly, it would be highly desirable to identify alternative, noncorrosive and weakly absorbing redox couples ${ }^{5-7}$. This will not only further boost up the PCE but also promote the practical implementation of this promising lowcost photovoltaic technology.

Recently, Wang et al. reported a promising disulfide/thiolate $\left(\mathrm{T}^{-} / \mathrm{T}_{2}\right)$ redox couple that has negligible absorption in the visible spectral range. Using this novel noncorrosive redox electrolyte in conjunction with a Z-907Na sensitized heterojunction, a PCE up to $6.4 \%$ was achieved under standard illumination conditions ${ }^{6}$. However, conventional planar platinum counter electrode showed poor catalytic activity towards this iodine-free redox couple, thus resulting in a poor fill factor (FF) and relatively moderate PCE. The phenomenon of a low FF with sulfur-functionalized redox mediators most likely results from the slow catalytic reduction of the oxidized species at the platinum counter electrode ${ }^{6,8,9}$. The slow electron transfer thus leads to a high interfacial charge transfer resistance limiting the FF of the device ${ }^{10}$. Therefore, it is imperative to seek suitable platinum-free catalysts that exhibit fast electron transfer kinetics for the disulfide-to-thiolate reduction, especially taking into account the low abundance $(0.0037 \mathrm{ppm})$ and high cost (US\$50/gram) of the platinum resources ${ }^{11}$. 
Additionally, semiconductor quantum dots (QDs) such as CdS, $\mathrm{CdSe}, \mathrm{PbS}$, InAs and InP constitute reliable alternative light absorbers $^{12,13}$ due to their intrinsic properties (i.e., size-dependent band gap $^{14}$, high extinction coefficients ${ }^{15}$, and large intrinsic dipole moment $\left.{ }^{16}\right)$. More importantly, demonstration of the multiple exciton generation by impact ionization in colloidal $\mathrm{QDs}^{17}$ could push the thermodynamic efficiency limit of these devices up to $44 \%{ }^{18}$ instead of the current $31 \%$ of the Shockley-Queisser detailed balance limit ${ }^{19}$. However, most QDs suffer from photodegradation when used in conjunction with the $\mathrm{I}^{-} / \mathrm{I}_{3}{ }^{-}$redox couple $e^{20}$. Consequently, quantum dot-sensitized solar cells (QDSCs) are often based on aqueous polysulfide electrolyte with minor use of the $\mathrm{Fe}(\mathrm{CN})_{6}{ }^{3-} / \mathrm{Fe}(\mathrm{CN})_{6}{ }^{4-}$ couple $^{21}$ and various cobalt-based electrolytes ${ }^{22}$ featuring notable attenuated corrosiveness, as well as, adjustable redox potential. Normally, in conjunction with such polysulfide electrolytes, $\mathrm{Pt}$ and other noble metals like $\mathrm{Au}$ are poor catalysts, and generate considerable overpotentials for the electrolyte regeneration. This is reflected in a reduction of the fill factors and thus PCE of the solar cell ${ }^{23}$. Therefore, alternative catalytic materials for polysulfide solution have been investigated such as $\mathrm{CoS}, \mathrm{Cu}_{2} \mathrm{~S}$, NiS, and carbon material $s^{24-26}$. However, low electrocatalytic activities were observed for carbon, $\mathrm{Au}$, and $\mathrm{Pt}$, followed by $\mathrm{NiS}$, while the best results were obtained for $\mathrm{CoS}$ and $\mathrm{Cu}_{2} \mathrm{~S}$ recently. Nevertheless, stability measurements in the presence of a photoanode indicated that $\mathrm{CoS}$ and $\mathrm{Cu}_{2} \mathrm{~S}$ electrodes may contaminate the electrolyte and subsequently the photoanode ${ }^{24}$. Therefore, it would be of high importance to identify alternative highly-active catalytic counter electrode materials for further progress of this polysulfide electrolytes widely utilized in QDSCs.

To this end, efforts have been made to replace Pt with abundant low-cost alternatives in these iodine-free electrolyte systems, including tungsten carbide embedded into the ordered mesoporous carbon $^{8}$, mesoporous carbon nanofibers ${ }^{27}$, niobium oxide ${ }^{28}, \mathrm{Cu}_{2} \mathrm{~S}$ reduced graphene oxide composite ${ }^{29}$, and $\mathrm{PbS}^{23}$. It is worth mentioning that carbon nanotube (CNT) has recently attracted numerous attentions as practical Pt-free counter electrodes material in iodine electrolyte based DSCs and exhibited reasonable performance ${ }^{30-33}$. Specifically, aligned multi-walled carbon nanotube (MWCNT) arrays have been widely used to replace the platinum counter electrodes to catalyze the reduction of triiodide in DSCs with the target of better performance and lower material $\operatorname{cost}^{34-39}$. In 2011, Peng's group developed a new fabrication method for aligned CNTs/polymer film as the electrode material ${ }^{36}$ and reported an efficiency of $6.6 \%{ }^{40}$. It is noted that Cheng's team successfully obtained a PCE of $6.05 \%$ in the iodine electrolyte system by utilizing a hybrid carbon structure, VACNTs grown on graphene paper ${ }^{38}$.

Single-walled carbon nanotubes (SWCNTs) can provide much higher surface area than MWCNTs due to their single graphitic layer structure and small diameter. Most recently, the efficiency of gelcoated SWCNTs-DSC reached $8 \%$ in conjunction with iodine electrolyte $^{35}$. According to the high surface area to volume ratio in the vertically aligned $3 \mathrm{D}$ structure, even higher available catalytic surface area can be achieved in the vertically aligned single-wall carbon nanotubes (VASWCNTs). Moreover, electrons can directly transfer through individual tubes, instead of hopping between the different carbon structures within this film. As the result, VASWCNTs have been applied as low-cost counter electrode material for DSCs in our recent work $^{32}$ and demonstrated comparable PCE with iodine electrolyte to the conventional Pt analogue. However, to the best of our knowledge, these promising three-dimensional electrocatalysts have not been employed in the iodine-free electrolyte up to date. Such results motivated us to explore the implementation of VASWCNTs as potential electrocatalyst for the aforementioned sulfide-mediated electrolytes.

Herein, we present for the first time, VASWCNTs that have been successfully transferred onto transparent conducting oxide glass and implemented as efficient low-cost, platinum-free counter electrode in iodine-free DSCs, featuring notably improved electrocatalytic activity toward thiolate/disulfide redox shuttle $e^{6}$ and aqueous polysulfide electrolyte over that of conventional planar Pt counter electrode. Impressively, The charge-transfer resistance $\left(R_{\mathrm{ct}}\right)$ of VASWCNTs-counter electrode is only $21.22 \Omega$ towards aqueous polysulfide electrolyte commonly applied in QDSCs. This value is several orders of magnitude lower than that of a Pt electrode. DSCs devices incorporating VASWCNTs counter electrodes and thiolate/disulfide redox electrolytes also show a significant gain in FF and short-circuit photocurrent density with respect to that of Pt electrodes.

\section{Results}

As depicted in Figure 1, high quality VASWCNTs arrays were firstly grown in a water-assisted thermal chemical vapor deposition (CVD) system at $750^{\circ} \mathrm{C}$ (Process 1 in Figure 1$)^{41}$, and then dry-transferred onto FTO glass substrates to produce robust counter electrodes (Process 2 and 3 in Figure 1). When compared with wet processing methods, the dry-transfer method is simple, reproducible and easy to control, offering high packing density of the obtained VASWCNTs arrays. Such VASWCNTs offer strong van Der Waals interfacial adhesion with a contacting surface, compared to the interaction with the growth substrate. According to this, a simple dry contact transfer can be achieved following the principle of the "gecko-effect" in a fully dry state ${ }^{42}$. Figure 2 a presents a representative cross-sectional scanning electron microscope (SEM) image of the as-grown VASWCNTs arrays with a length around $30 \mu \mathrm{m}$, demonstrating the vertical alignment of these SWCNTs. As depicted in Figure 2b, following the drytransfer process, the lengths of the SWCNTs arrays are observed to be approximately $6 \mu \mathrm{m}$ due to the compression by an applied shear-force placed on the SWCNTs arrays, which is in line with our previous study of the compression effect on the VASWCNTs arrays' lengths ${ }^{32}$. Top surface morphology from Figure $2 c$ clearly demonstrates the large interstices around the CNTs bundles. Such

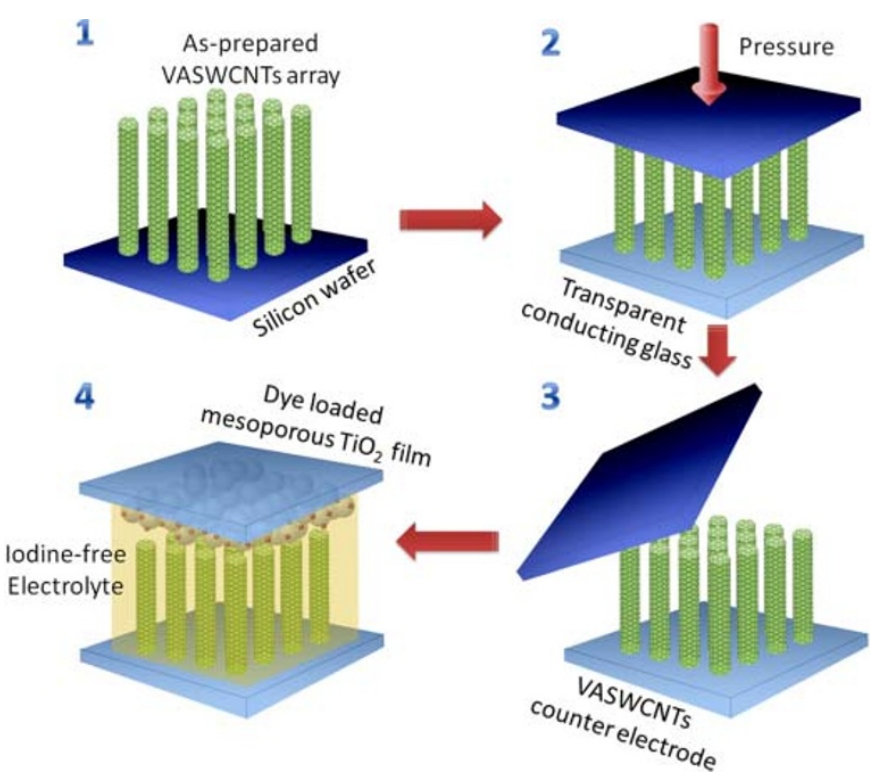

Figure $1 \mid$ Process flow of preparing the VASWCNTs-DSCs. Pre-etched VASWCNTs on silicon substrate (Process 1) were flipped on top of the FTO-glass, and then a force was loaded onside the silicon top (Process 2). After simply removing the silicon wafer (Process 3), the VASWCNTs were transferred onto the FTO-glass substrate. Finally, the VASWCNTs-DSC (Process 4) was successfully assembled by utilizing this new counter electrode. 

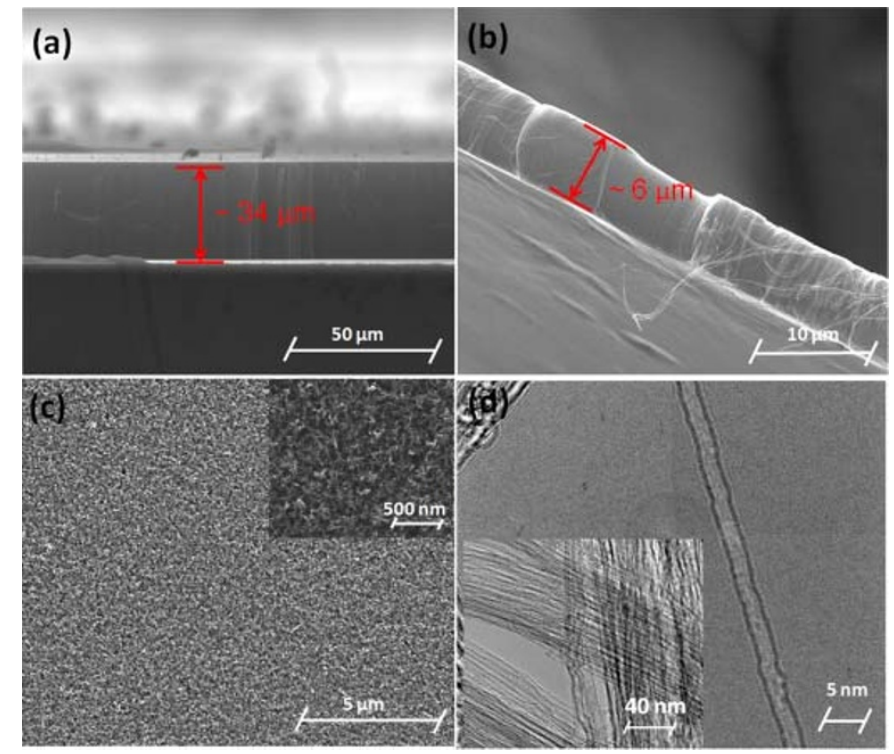

Figure $2 \mid$ Representative cross-sectional SEM images of as-grown (a), transferred (b), top morphological (c) and TEM (d) images of VASWCNTs. Insets of (c) and (d) show the magnified topomorphology and panoramic TEM image.

a geometrical structure of the VASWCNTs possesses doublefunctional merits of direct electron transport through individual vertically aligned tubes as well as rapid redox diffusion in the open structure around tubes. Figure $2 \mathrm{~d}$ shows a typical transmission electron microscopy (TEM) image of the SWCNTs material, after sonication in ethanol and drop casting onto a TEM grid. It is evident from these images that SWCNTs have a diameter range of $2 \sim 4 \mathrm{~nm}$, giving rise to a significant high surface area of the CNTs arrays. Due to its repeatability and simplicity, the dry contact transfer printing is highly scalable. This technique can be applied in a roll-to-roll, or large-scale industrial manufacturing settings.

Raman spectroscopy was further applied to characterize the quality of the VASWCNTs arrays. From the Raman characterization (633 nm excitation) of Figure 3, it is obvious that the VASWCNTs are of high quality with a G/D ratio around 9 . This suggests a very low amount of defects, such as amorphous carbon in the nanotubes, as indicated by the red circles in Figure S1. At the same time, radial breathing modes (RBM) can be observed from the Raman spectra of the materials.

To assess the potential applications of the VASWCNTs counter electrodes in DSCs, devices were assembled with N719 sensitized

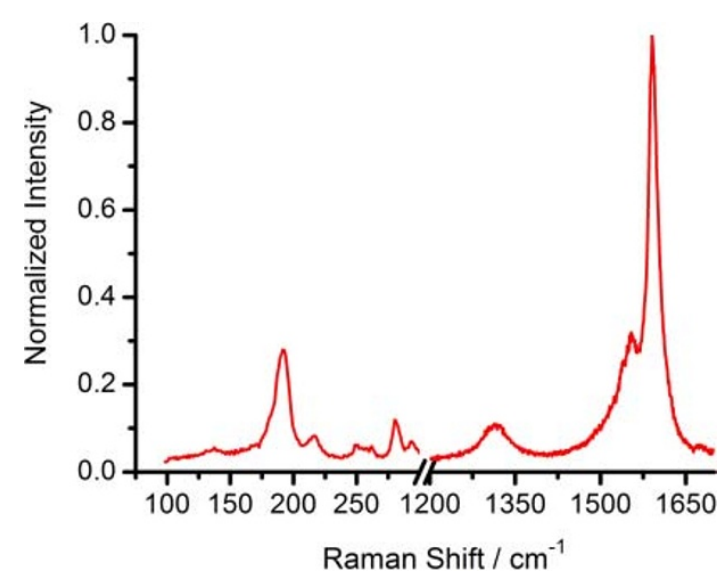

Figure 3 Raman spectra of VASWCNTs electrodes (633 $\mathrm{nm}$ excitation).
$\mathrm{TiO}_{2}$ electrodes with a thickness of $14 \mu \mathrm{m}$ and an active area of $0.16 \mathrm{~cm}^{2}$ in conjunction with the VASWCNTs electrodes (Process 4 in Figure 1) or conventional sputtered Pt counter electrodes. Figure 4 demonstrates the characteristic $J$ - $V$ performance for devices with VASWCNTs and reference Pt counter electrodes measured under Air Mass 1.5G illumination and the detailed photovoltaic parameters are tabulated in Table 1. Device A with Pt electrode in conjunction with $\mathrm{T}^{-} / \mathrm{T}_{2}$ electrolyte exhibits an open-circuit voltage $\left(V_{\mathrm{oc}}\right)$ of $612 \mathrm{mV}$, a short-circuit photocurrent density $\left(J_{\mathrm{sc}}\right)$ of $10.26 \mathrm{~mA} \mathrm{~cm}^{-2}$, an FF of 0.56 , yielding an overall PCE of $3.49 \%$. It is remarkable that device $\mathrm{B}$ with VASWCNTs displays a $J_{\mathrm{sc}}$ enhancement up to $12.66 \mathrm{~mA} \mathrm{~cm}^{-2}$, a considerable increase in $\mathrm{FF}$ up to 0.68 , and produces a PCE of 5.25\%. Apparently, the enhancement of the PCE is directly originated from the $J_{\mathrm{sc}}$ and FF increase.

\section{Discussion}

It is important to note that the FF of a DSC device is attenuated by the total series resistance of the cell, including the sheet resistances of the substrate and counter electrode, electron transport resistance through the photoanode, ion transport resistance, and $R_{\mathrm{ct}}$ at the counter electrode ${ }^{43}$. Therefore, the FF enrichment in the current work is mainly a result of the superior electrocatalytic activity of the VASWCNTs and consequent significantly reduced chargetransfer resistance across the counter electrode/electrolyte interface. Significant improvement in charge transfer at the counter electrode/ electrolyte interface not only reduces internal resistances, but also attenuates the recombination rates and concentration gradients in the electrolyte, which have been proved to affect $J_{\text {sc }}$ strongly ${ }^{23}$. The obtained FF and PCE is of great advantage when compared to that with other catalyst materials, such as tungsten carbide embedded ordered mesoporous carbon ${ }^{8}$, as well as graphite ${ }^{9}$. Efforts to further enhance the PCE are currently in progress in our laboratory, which include optimizing the photoanode structure and employment of other sensitizers with strong hydrophobicity to inhibit charge recombination between the photoelectrode and electrolyte.

The electrocatalytic activity of the as-fabricated VASWCNTs counter electrodes towards thiolate/disulfide redox shuttle ${ }^{6}$ was first investigated by electrochemical impedance spectroscopy (EIS $)^{44,45}$. Figure 5a presents the corresponding Nyquist spectra of symmetrical dummy cells with VASWCNTs counter electrodes and conventional

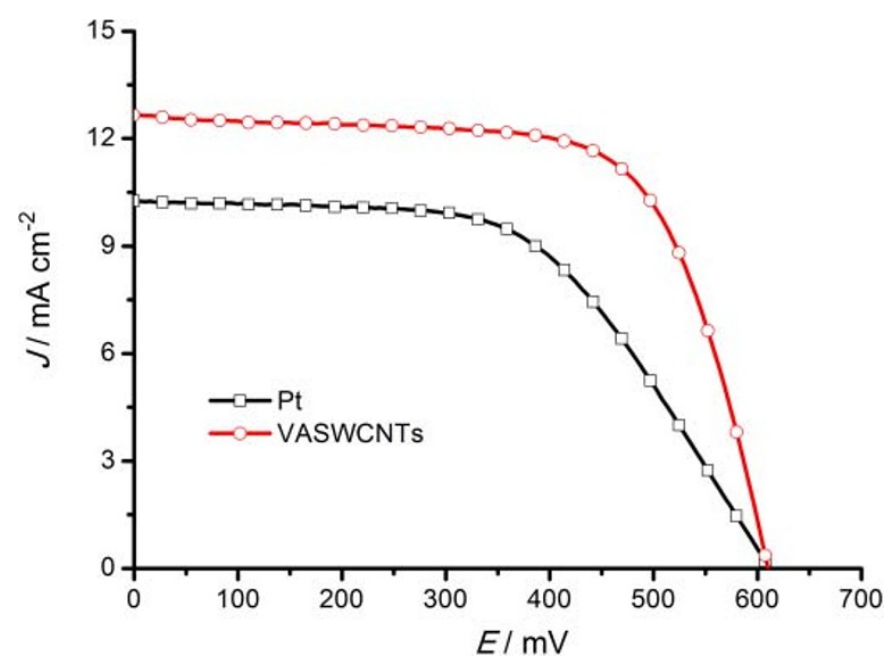

Figure $4 \mid$ Photocurrent-voltage characteristics of DSCs using VASWCNTs or Pt counter electrodes under 1 sun illumination (AM $1.5 \mathrm{G}, 100 \mathrm{~mW} \mathrm{~cm}^{-2}$ ). The electrolyte was comprised of $0.4 \mathrm{M} \mathrm{T}^{-}, 0.4 \mathrm{M}$ $\mathrm{T}_{2}, 0.5 \mathrm{M}$ 4-tertbutylpyridine, and $0.05 \mathrm{M} \mathrm{LiOCl}_{4}$ in acetonitrile: ethylene carbonate $(6: 4, \mathrm{~V}: \mathrm{V})$. 
Table 1 | EIS parameters fitted from the equivalent circuit and corresponding photovoltaic performance of DSCs with different counter electrodes in conjunction with $\mathrm{T}^{-} / \mathrm{T}_{2}$ electrolyte under 1 sun illumination $\left(\mathrm{AM} 1.5 \mathrm{G}, 100 \mathrm{~mW} \mathrm{~cm}^{-2}\right)^{[\mathrm{a}]}$

\begin{tabular}{|c|c|c|c|c|c|c|c|}
\hline Electrodes & $R_{\mathrm{s}}[\Omega]$ & $R_{\mathrm{ct}}[\Omega]$ & $C_{\text {dil }}[\mathrm{F}]$ & $V_{\text {oc }}[\mathrm{mV}]$ & $J_{\mathrm{sc}}\left[\mathrm{mA} \mathrm{cm}^{-2}\right]$ & $\mathrm{FF}$ & PCE[\%] \\
\hline $\mathrm{Pt}$ & $26.08 \pm 0.12$ & $76.28 \pm 2.37$ & $(4.93 \pm 0.24) \mathrm{E}-8$ & $612 \pm 4$ & $10.26 \pm 0.29$ & $0.56 \pm 0.02$ & $3.49 \pm 0.17$ \\
\hline VASWCNTs & $25.67 \pm 0.08$ & $12.66 \pm 1.85$ & $(4.60 \pm 0.43) \mathrm{E}-6$ & $610 \pm 3$ & $12.66 \pm 0.35$ & $0.68 \pm 0.03$ & $5.25 \pm 0.23$ \\
\hline
\end{tabular}

$[\mathrm{a}] R_{\mathrm{s}}=$ series resistance; $R_{\mathrm{ct}}=$ charge-transfer resistance; $C_{\mathrm{dl}}=$ double-layer capacitance; $V_{\mathrm{oc}}=$ open-circuit voltage; $J_{\mathrm{sc}}=$ short-circuit photocurrent density; $F F=$ fill factor; $P C E=$ power conversion efficiency. Values presented here are the average of at least three samples.

sputtered Pt counter electrodes as the reference. It is generally recognized that in the order of increasing frequency, the response can be attributed to the Warburg diffusion processes in the electrolyte and charge transfer at the counter electrode/electrolyte interface ${ }^{45}$. Apparently, incorporation of the VASWCNTs promotes a significantly reduced diameter of the high-frequency semicircles. By fitting with the Randles equivalent circuit shown in Figure S2, the $R_{\mathrm{ct}}$ of the VASWCNTs as shown in Table 1 is only $12.66 \Omega$, a value almost sixfold lower than that of conventional Pt counter electrode (76.28 $\Omega$ ) under similar measurement conditions, highlighting the superior electrocatalytic activity of the VASWCNTs towards thiolate/disulfide redox couples. Furthermore, obvious high-frequency shift of the characteristic response peaks in the high-frequency regime of Bode plot shown in Figure 5b demonstrates a faster electron transfer at the interface between VASWCNTs and electrolyte with respect to conventional Pt counter electrode. In addition, it is also observed that the VASWCNTs electrodes exhibit a notably larger double-layer capacitance $\left(C_{\mathrm{dl}}\right)$ than the planar Pt electrodes do, suggesting a higher specific surface area in the former counter electrodes. This is in
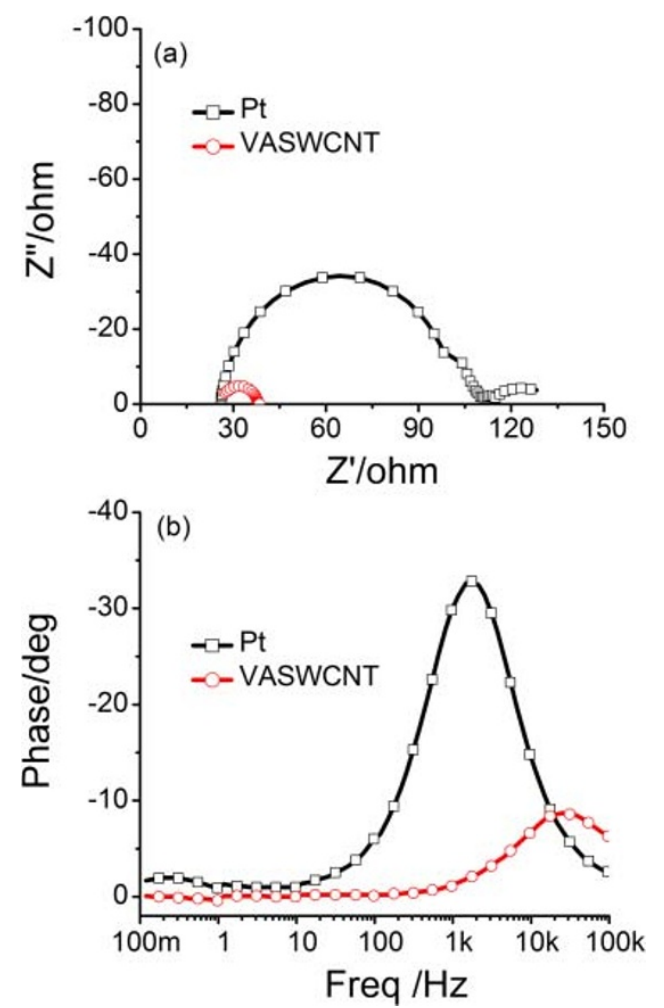

Figure 5 Corresponding Nyquist plots (a) and Bode plots (b) of the symmetrical dummy cell towards thiolate/disulfide electrolyte with a recipe of $0.4 \mathrm{M} \mathrm{T}^{-}, 0.4 \mathrm{M} \mathrm{T}_{2}, 0.5 \mathrm{M}$ 4-tertbutylpyridine, and $0.05 \mathrm{M}$ $\mathrm{LiOCl}_{4}$ in acetonitrile: ethylene carbonate $(6: 4, \mathrm{~V}: \mathrm{V})$. The frequency range was set from $100 \mathrm{mHz}$ to $1 \mathrm{MHz}$, and the amplitude of the alternating current was set to $10 \mathrm{mV}$ in impedance measurements. agreement with the results from other nanostructured counter electrodes with a high porosity ${ }^{46,47}$. Noteworthy is that our VASWCNTs do not increase the redox diffusion resistance in the electrolyte, probably arising from the relative open structure as characterized by the SEM image in Figure $2 c$.

The superior electrocatalytic activity of VASWCNTs electrodes towards $\mathrm{T}^{-} / \mathrm{T}_{2}$ electrolyte inspired us to the implementation of this encouraging counter electrode in other electrolyte systems, such as the aqueous polysulfide electrolyte commonly applied in QDSCs. Similar to the aforementioned experimental conditions, symmetrical dummy cells were fabricated in combination with the aqueous polysulfide electrolyte with a recipe of $1 \mathrm{M}$ sulfur, $1 \mathrm{M} \mathrm{Na}_{2} \mathrm{~S} \cdot 9 \mathrm{H}_{2} \mathrm{O}$, and $0.1 \mathrm{M} \mathrm{NaOH}$ in deionized water. Figure 6 displays the corresponding Nyquist plots (a), Bode plots (b) and Tafel curves (c) of the symmetrical dummy cells measured under the similar conditions. Dramatic reduction in the $R_{\mathrm{ct}}$ of VASWCNTs electrode is observed in comparison with that of Pt electrode as summarized in Table S1. In parallel with previous studies on the catalytic activity for polysulfide solutions ${ }^{24-26,48-50}$, planar Pt electrode exhibits a huge $R_{\mathrm{ct}}$ in the order of $10^{5} \Omega$, mainly due to the poor catalytic activity. Encouragingly, utilization of the VASWCNTs greatly enhances the electrocatalytic activity, yielding a small $R_{\mathrm{ct}}$ value of only $21.22 \Omega$, as seen from the inset of Figure 6a. To the best of our knowledge, such an $R_{\mathrm{ct}}$ value is the lowest ever seen in the polysulfide electrolyte, even compared with recently reported $\mathrm{PbS}^{23}$ and $\mathrm{Cu}_{2} \mathrm{~S}^{19}$ counter electrodes. Complementally, significant high-frequency shift of the characteristic response peaks in the Bode plot shown in Figure $6 \mathrm{~b}$, further confirms the superior catalytic activity of the VASWCNTs electrodes to polysulfide electrolytes.

Tafel polarization measurement was further employed to study the interfacial charge-transfer properties of the polysulfide redox couples on the counter electrode surface. Figure $6 \mathrm{c}$ shows the logarithmic current density as a function of applied voltage of the symmetrical dummy cells. The exchange current density $\left(J_{0}\right)$ is obtained as the intercept of the extrapolated linear region of the curve when the overpotential is zero. In the curve at high potential (horizontal part), the limiting diffusion current density $\left(J_{\text {lim }}\right)$ can be derived. These two parameters are closely related with the electrochemical activity of the catalyst $^{51}$. Evidently, the anodic and cathodic branches of the VASWCNTs electrodes exhibit an obvious larger slope than the conventional Pt counter electrodes do, indicating the presence of a large exchange current density $\left(J_{0}\right)$ on the electrode surfaces. Moreover, a higher $J_{\lim }$ is noted in conjunction with VASWCNTs counter electrode, which suggests a higher diffusion coefficient of the polysufide redox couple in the electrolyte compared to that of $\mathrm{Pt}$ electrode according to Equation (1):

$$
D=\frac{l}{2 n F C} J_{\lim }
$$

where $D$ is the diffusion coefficient of the polysulfide, $l$ is the electrolyte thickness, $n$ is the number of electrons involved in the reduction of disulphide at the counter electrode, $F$ is the Faraday constant, and $C$ is the polysulfide concentration ${ }^{52}$. These results confirm that the catalytic activity towards polysulfide redox shuttle of VASWCNTs is superior to that of conventional Pt counter electrodes. Furthermore, studies on the photovoltaic implementation of 

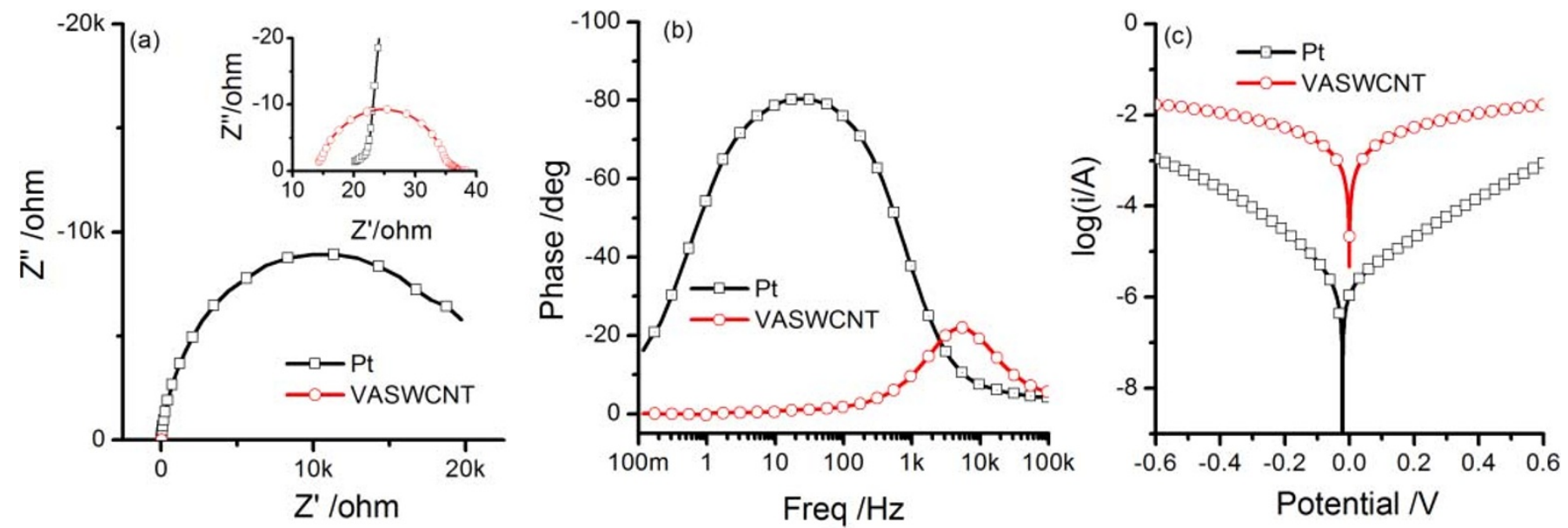

Figure 6 Corresponding Nyquist plots (a), Bode plots (b) and Tafel curves (c) of the symmetrical dummy cell towards aqueous polysulfide electrolyte with a recipe of $1 \mathrm{M}$ sulfur, $1 \mathrm{M} \mathrm{Na}_{2} \mathrm{~S} 9 \mathrm{H}_{2} \mathrm{O}$, and $0.1 \mathrm{M} \mathrm{NaOH}$ in deionized water. The frequency range was set from $100 \mathrm{mHz}$ to $1 \mathrm{MHz}$, and the amplitude of the alternating current was set to $10 \mathrm{mV}$ in the impedance measurements. Tafel measurements were performed with a scanning rate of $10 \mathrm{mV} \mathrm{s}^{-1}$.

VASWCNTs counter electrodes in QDSCs are in progress in our laboratory.

In summary, we have developed VASWCNTs as efficient low-cost counter electrode material in iodine-free DSCs, featuring notably improved electrocatalytic activity towards thiolate/disulfide redox shuttle. Spectacularly, device in conjunction with VASWCNTs counter electrode demonstrates a high FF of 0.68 and PCE up to $5.25 \%$, which is significant higher than 0.56 and $3.49 \%$ for that with a conventional Pt counter electrode. Moreover, in QDSCs system, the $R_{\mathrm{ct}}$ of VASWCNTs counter electrode is as low as $21.22 \Omega$ towards aqueous polysulfide electrolyte. This is several orders of magnitude lower than that for $\mathrm{Pt}$ electrode. In comparison with alternative counter electrode materials previous reported such as $\mathrm{Au}, \mathrm{CoS}$, $\mathrm{Cu}_{2} \mathrm{~S}$ or $\mathrm{PbS}$, to the best of our knowledge, such a $R_{\mathrm{ct}}$ value is the lowest ever seen in the polysulfide electrolyte system. Therefore, the highly catalytic VASWCNTs counter electrodes are believed to be a versatile candidate for further improvement of the PCE of other iodine-free redox couple based DSCs and polysulfide electrolyte based QDSCs.

\section{Methods}

Similar to our previous work ${ }^{32}, 10 \mathrm{~nm}$ thick $\mathrm{Al}_{2} \mathrm{O}_{3}$ film and subsequent $1 \mathrm{~nm}$ thick Fe film are deposited onto $\mathrm{Si}$ substrate. VASWCNTs growth was carried out in a waterassisted thermal CVD system at $750^{\circ} \mathrm{C}$. After a quick cooling treatment, the postgrowth $\mathrm{H}_{2} / \mathrm{H}_{2} \mathrm{O}$ vapor etch took $3 \sim 5 \mathrm{~min}$ at $750^{\circ} \mathrm{C}$. This etch would release the chemical bonds between the nanotubes and the catalyst particles. And then the VASWCNTs were transferred onto FTO substrate by simple dry contact transfer printing ${ }^{53}$.

Conventional sputtered Pt counter electrode was prepared by an ion sputtering coater (SBC-12, KYKY Technology Development Ltd., China) for comparison. The thickness of the obtained Pt film was approximately $50 \mathrm{~nm}$. DSCs devices were fabricated according to our previous reports ${ }^{54}$. Briefly, $\mathrm{TiO}_{2}$ paste composed of $18 \%$ commercial P25 nanoparticles and 9\% ethyl cellulose in terpineol was used to screen printing porous $\mathrm{TiO}_{2}$ films (approximately $14 \mu \mathrm{m}$ thick) on FTO substrate (sheet resistance: $12 \Omega$ /square, Nippon Sheet Glass, Co., Ltd.). The obtained $\mathrm{TiO}_{2}$ electrodes were dipped into dry ethanol solution containing $5 \times 10^{-4} \mathrm{M}$ cis-bis(isothiocyanato) bis (2, 2' -bipyridyl-4,4' -dicarboxylato) ruthenium(II) bistetrabuyl-ammonium (N719, ruthenium TBA535, Solaronix, Switzerland) for $20 \mathrm{~h}$. The dye-sensitized $\mathrm{TiO}_{2}$ electrodes, VASWCNTs and Pt counter electrodes were assembled to form DSCs by sandwiching a redox couple $\left(\mathrm{T}^{-} / \mathrm{T}_{2}\right)$ electrolyte solution composed of $0.4 \mathrm{M}$ $\mathrm{T}^{-}, 0.4 \mathrm{M} \mathrm{T}_{2}, 0.5 \mathrm{M}$ 4-tertbutylpyridine, and $0.05 \mathrm{M} \mathrm{LiOCl}_{4}$ in acetonitrile:ethylene carbonate $=6: 4, \mathrm{~V}: \mathrm{V})$. The organic redox couple $\left(\mathrm{T}^{-} / \mathrm{T}_{2}\right)$ was synthesized by the literature method $^{6}$. Aqueous polysulfide electrolyte was composed of $1 \mathrm{M}$ sulfur, $1 \mathrm{M}$ $\mathrm{Na}_{2} \mathrm{~S} 9 \mathrm{H}_{2} \mathrm{O}$, and $0.1 \mathrm{M} \mathrm{NaOH}$ in deionized water.

The morphologies of the obtained VASWCNTs were characterized by field emission scanning electron microscopy (FEI Quanta 400 ESEM, FEI, USA) and transmission electron microscopy (TEM, JEOL 2010, JEOL, Japan). Raman characterization ( $633 \mathrm{~nm}$ excitation) was performed by a Renishaw inVia Raman Microscope. To assess the corresponding catalytic performance of different counter electrodes, the $R_{\mathrm{ct}}$ across the counter electrode/electrolyte interface was investigated by means of EIS with the symmetrical thin-layer cells method. The frequency range was set from $100 \mathrm{mHz}$ to $1 \mathrm{MHz}$, and the amplitude of the alternating current was set to $10 \mathrm{mV}$. Tafel measurements were performed with a scanning rate of $10 \mathrm{mV} \mathrm{s}^{-1}$ in the potential range of $0.6 \mathrm{~V}$ to $-0.6 \mathrm{~V}$. The photocurrent-voltage $(J-V)$ characteristics of DSCs were measured with a digital source meter (2400, Keithley Instruments, USA) under AM 1.5G illumination $\left(100 \mathrm{~mW} \mathrm{~cm}^{-2}\right)$, which was realized by a solar simulator (91192, Oriel, USA, calibrated with a standard crystalline silicon solar cell)

1. Lewis, N. S. \& Nocera, D. G. Powering the planet: Chemical challenges in solar energy utilization. Proc. Natl. Acad. Sci. USA 103, 15729-15735 (2006).

2. Oregan, B. \& Gätzel, M. A Low-Cost, High-Efficiency Solar-Cell Based on DyeSensitized Colloidal TiO 2 Films. Nature 353, 737-740 (1991).

3. Chiba, Y. et al. Dye-sensitized solar cells with conversion efficiency of $11.1 \%$. Jpn. J. Appl. Phys. 2 45, L638-L640 (2006).

4. Boschloo, G. \& Hagfeldt, A. Characteristics of the Iodide/Triiodide Redox Mediator in Dye-Sensitized Solar Cells. Acc. Chem. Res. 42, 1819-1826 (2009).

5. Yanagida, S., Yu, Y. H. \& Manseki, K. Iodine/Iodide-Free Dye-Sensitized Solar Cells. Acc. Chem. Res. 42, 1827-1838 (2009).

6. Wang, M. K. et al. An organic redox electrolyte to rival triiodide/iodide in dyesensitized solar cells. Nat. Chem. 2, 385-389 (2010).

7. Daeneke, T. et al. High-efficiency dye-sensitized solar cells with ferrocene-based electrolytes. Nat. Chem. 3, 211-215 (2011).

8. Wang, L., Wu, M. X., Gao, Y. R. \& Ma, T. L. Highly catalytic counter electrodes for organic redox couple of thiolate/disulfide in dye-sensitized solar cells. Appl. Phys. Lett. 98, 221102 (2011).

9. Wu, H. W. et al. Graphite and platinum's catalytic selectivity for disulfide/thiolate $\left(\mathrm{T}_{2} / \mathrm{T}^{-}\right)$and triiodide/iodide $\left(\mathrm{I}_{3}{ }^{-} / \mathrm{I}^{-}\right)$. J. Mater. Chem. 21, 14815-14820 (2011).

10. Han, L. Y. et al. Improvement of efficiency of dye-sensitized solar cells by reduction of internal resistance. Appl. Phys. Lett. 86, 213501 (2005).

11. Ahmad, S., Yum, J. H., Butt, H. J., Nazeeruddin, M. K. \& Grätzel, M. Efficient Platinum-Free Counter Electrodes for Dye-Sensitized Solar Cell Applications. ChemPhysChem 11, 2814-2819 (2010).

12. Nozik, A. J. et al. Semiconductor Quantum Dots and Quantum Dot Arrays and Applications of Multiple Exciton Generation to Third-Generation Photovoltaic Solar Cells. Chem. Rev. 110, 6873-6890 (2010).

13. Kongkanand, A., Tvrdy, K., Takechi, K., Kuno, M. \& Kamat, P. V. Quantum dot solar cells. Tuning photoresponse through size and shape control of $\mathrm{CdSe}-\mathrm{TiO}_{2}$ architecture. J. Am. Chem. Soc. 130, 4007-4015 (2008).

14. Yu, W. W., Qu, L. H., Guo, W. Z. \& Peng, X. G. Experimental determination of the extinction coefficient of CdTe, CdSe, and CdS nanocrystals. Chem. Mater. 15 2854-2860 (2003).

15. Sun, J. J. \& Goldys, E. M. Linear absorption and molar extinction coefficients in direct semiconductor quantum dots. J. Phys. Chem. C 112, 9261-9266 (2008).

16. Hanewinkel, B., Knorr, A., Thomas, P. \& Koch, S. W. Optical near-field response of semiconductor quantum dots. Phys. Rev. B 55, 13715-13725 (1997).

17. Sambur, J. B., Novet, T. \& Parkinson, B. A. Multiple Exciton Collection in a Sensitized Photovoltaic System. Science 330, 63-66 (2010).

18. Klimov, V. I. Mechanisms for photogeneration and recombination of multiexcitons in semiconductor nanocrystals: Implications for lasing and solar energy conversion. J. Phys. Chem. B 110, 16827-16845 (2006).

19. Shockley, W. \& Queisser, H. J. Detailed Balance Limit of Efficiency of P-N Junction Solar Cells. J. Appl. Phys. 32, 510-519 (1961). 
20. Shalom, M., Dor, S., Ruhle, S., Grinis, L. \& Zaban, A. Core/CdS Quantum Dot/ Shell Mesoporous Solar Cells with Improved Stability and Efficiency Using an Amorphous $\mathrm{TiO}_{2}$ Coating. J. Phys. Chem. C 113, 3895-3898 (2009).

21. Tachibana, Y., Umekita, K., Otsuka, Y. \& Kuwabata, S. Charge Recombination Kinetics at an in Situ Chemical Bath-Deposited CdS/Nanocrystalline $\mathrm{TiO}_{2}$ Interface. J. Phys. Chem. C 113, 6852-6858 (2009).

22. Lee, H. J. et al. CdSe quantum dot-sensitized solar cells exceeding efficiency $1 \%$ at full-sun intensity. J. Phys. Chem. C 112, 11600-11608 (2008).

23. Tachan, Z. et al. PbS as a Highly Catalytic Counter Electrode for Polysulfide-Based Quantum Dot Solar Cells. J. Phys. Chem. C 115, 6162-6166 (2011).

24. Hodes, G., Manassen, J. \& Cahen, D. Electrocatalytic Electrodes for the Polysulfide Redox System. J. Electrochem. Soc. 127, 544-549 (1980).

25. Weidman, M. C., Esposito, D. V., Hsu, I. J. \& Chen, J. G. Electrochemical Stability of Tungsten and Tungsten Monocarbide (WC) Over Wide $\mathrm{pH}$ and Potential Ranges. J. Electrochem. Soc. 157, F179-F188 (2010).

26. Fan, S. Q. et al. Hierarchical nanostructured spherical carbon with hollow core/ mesoporous shell as a highly efficient counter electrode in CdSe quantum-dotsensitized solar cells. Appl. Phys. Lett. 96063501 (2010).

27. Fang, B. et al. Facile synthesis of open mesoporous carbon nanofibers with tailored nanostructure as a highly efficient counter electrode in CdSe quantum-dotsensitized solar cells. J. Mater. Chem. 21, 8742-8748 (2011).

28. Lin, X., Wu, M. X., Wang, Y. D., Hagfeldt, A. \& Ma, T. L. Novel counter electrode catalysts of niobium oxides supersede Pt for dye-sensitized solar cells. Chem. Commun. 47, 11489-11491 (2011)

29. Radich, J. G., Dwyer, R. \& Kamat, P. V. Cu 2 S Reduced Graphene Oxide Composite for High-Efficiency Quantum Dot Solar Cells. Overcoming the Redox Limitations of $\mathrm{S}^{2-} / \mathrm{S}_{\mathrm{n}}{ }^{2-}$ at the Counter Electrode. J. Phys. Chem. Lett. 2, 2453-2460 (2011).

30. Han, J., Kim, H., Kim, D. Y., Jo, S. M. \& Jang, S. Y. Water-Soluble PolyelectrolyteGrafted Multiwalled Carbon Nanotube Thin Films for Efficient Counter Electrode of Dye-Sensitized Solar Cells. ACS Nano 4, 3503-3509 (2010).

31. Nam, J. G., Park, Y. J., Kim, B. S. \& Lee, J. S. Enhancement of the efficiency of dyesensitized solar cell by utilizing carbon nanotube counter electrode. Scr. Mater. 62, 148-150 (2010).

32. Dong, P. et al. Vertically Aligned Single-Walled Carbon Nanotubes as Low-cost and High Electrocatalytic Counter Electrode for Dye-Sensitized Solar Cells. ACS Appl. Mater. Interfaces 3, 3157-3161 (2011).

33. Hsieh, C. T., Yang, B. H. \& Lin, J. Y. One- and two-dimensional carbon nanomaterials as counter electrodes for dye-sensitized solar cells. Carbon 49 3092-3097 (2011).

34. Han, J., Kim, H., Kim, D. Y., Jo, S. M. \& Jang, S. Y. Water-soluble polyelectrolytegrafted multiwalled carbon nanotube thin films for efficient counter electrode of dye-sensitized solar cells. ACS Nano 4, 3503-3509 (2010).

35. Mei, X., Cho, S. J., Fan, B. \& Ouyang, J. High-performance dye-sensitized solar cells with gel-coated binder-free carbon nanotube films as counter electrode. Nanotechnology 21, 395202 (2010)

36. Huang, S. Q. et al. A New and General Fabrication of an Aligned Carbon Nanotube/Polymer Film for Electrode Applications. Adv. Mater. 23, 4707-4710 (2011).

37. Lee, K. S., Lee, W. J., Park, N. G., Kim, S. O. \& Park, J. H. Transferred vertically aligned $\mathrm{N}$-doped carbon nanotube arrays: use in dye-sensitized solar cells as counter electrodes. Chem Commun (Camb) 47, 4264-4266 (2011)

38. Li, S. S. et al. Vertically Aligned Carbon Nanotubes Grown on Graphene Paper as Electrodes in Lithium-Ion Batteries and Dye-Sensitized Solar Cells. Adv. Energy Mater. 1, 486-490 (2011).

39. Yang, Z. et al. Aligned carbon nanotube sheets for the electrodes of organic solar cells. Adv. Mater. 23, 5436-5439 (2011).

40. Yang, Z. B. et al. Aligned Carbon Nanotube Sheets for the Electrodes of Organic Solar Cells. Adv. Mater. 23, 5436-5439 (2011).

41. Suzuki, K., Yamaguchi, M., Kumagai, M. \& Yanagida, S. Application of carbon nanotubes to counter electrodes of dye-sensitized solar cells. Chem. Lett. 32, 2829 (2003).

42. Kamperman, M., Kroner, E., del Campo, A., McMeeking, R. M. \& Arzt, E. Functional Adhesive Surfaces with "Gecko" Effect: The Concept of Contact Splitting. Adv. Eng. Mater. 12, 335-348 (2010).
43. Hamann, T. W., Jensen, R. A., Martinson, A. B. F., Van Ryswyk, H. \& Hupp, J. T. Advancing beyond current generation dye-sensitized solar cells. Energy Environ. Sci. 1, 66-78 (2008)

44. Hauch, A. \& Georg, A. Diffusion in the electrolyte and charge-transfer reaction at the platinum electrode in dye-sensitized solar cells. Electrochim. Acta 46, 34573466 (2001).

45. Wang, Q. et al. Characteristics of high efficiency dye-sensitized solar cells. J. Phys. Chem. B 110, 25210-25221 (2006).

46. Lee, W. J., Ramasamy, E., Lee, D. Y. \& Song, J. S. Efficient Dye-Sensitized Cells with Catalytic Multiwall Carbon Nanotube Counter Electrodes. ACS Appl. Mater. Interfaces 1, 1145-1149 (2009).

47. Joshi, P. et al. Electrospun Carbon Nanofibers as Low-Cost Counter Electrode for Dye-Sensitized Solar Cells. ACS Appl. Mater. Interfaces 2, 3572-3577 (2010).

48. Gimenez, S. et al. Energy transfer versus charge separation in hybrid systems of semiconductor quantum dots and $\mathrm{Ru}$-dyes as potential co-sensitizers of $\mathrm{TiO}_{2}$-based solar cells. J. Appl. Phys. 110014314 (2011).

49. Kiyonaga, T., Akita, T. \& Tada, H. Au nanoparticle electrocatalysis in a photoelectrochemical solar cell using CdS quantum dot-sensitized $\mathrm{TiO}_{2}$ photoelectrodes. Chem. Commun. 2011-2013 (2009).

50. Yang, Z. S., Chen, C. Y., Liu, C. W. \& Chang, H. T. Electrocatalytic sulfur electrodes for CdS/CdSe quantum dot-sensitized solar cells. Chem. Commun. 46, 5485-5487 (2010).

51. Wu, M. X., Lin, X. A., Hagfeldt, A. \& Ma, T. L. Low-Cost Molybdenum Carbide and Tungsten Carbide Counter Electrodes for Dye-Sensitized Solar Cells. Angew. Chem. Int. Ed. 50, 3520-3524 (2011).

52. Zakeeruddin, S. M. \& Grätzel, M. Solvent-Free Ionic Liquid Electrolytes for Mesoscopic Dye-Sensitized Solar Cells. Adv. Funct. Mater. 19, 2187-2202 (2009).

53. Pint, C. L. et al. Dry Contact Transfer Printing of Aligned Carbon Nanotube Patterns and Characterization of Their Optical Properties for Diameter Distribution and Alignment. ACS Nano 4, 1131-1145 (2010).

54. Hao, F., Lin, H., Zhou, C., Liu, Y. Z. \& Li, J. B. Bifunctional single-crystalline rutile nanorod decorated heterostructural photoanodes for efficient dye-sensitized solar cells. Phys. Chem. Chem. Phys. 13, 15918-15924 (2011).

\section{Acknowledgments}

Financial Supports from the National High Technology Research and Development Program of China (863 Program, 2011AA050522), the International Cooperation S\&T Cooperation Program of China (2010DFA64360), and the Welch Foundation (C-1716) are greatly appreciated. The authors thank Dr Y. Zhu for help with catalyst preparation and TEM imaging.

\section{Author contribution statement:}

F.H. and P.D. conceived the experiments. P.D., J.Z. and R.H.H. fabricated the carbon nanotube arrays. P.E.L. helped the TEM observation. F.H. and Y.Z. synthesized the disulfide redox couples. F.H. performed the electrochemical and photovoltaic experiments and processing of the data. F.H., P.D., J.L. and H.L. wrote the paper. All authors contributed to the discussion of the results and reviewed the manuscript

\section{Additional information}

Supplementary information accompanies this paper at http://www.nature.com/ scientificreports

Competing financial interests: The authors declare no competing financial interests.

License: This work is licensed under a Creative Commons

Attribution-NonCommercial-ShareAlike 3.0 Unported License. To view a copy of this license, visit http://creativecommons.org/licenses/by-nc-sa/3.0/

How to cite this article: Hao, F. et al. High Electrocatalytic Activity of Vertically Aligned Single-Walled Carbon Nanotubes towards Sulfide Redox Shuttles. Sci. Rep. 2, 368; DOI:10.1038/srep00368 (2012) 